\title{
'Evaluating an Advance Care Planning Curriculum: a Lecture, a Game, a Patient, and an Essay'-a medical student's perspective
}

\author{
Emma Mittelman ${ }^{1}$ (D) Rajiv Ark ${ }^{1}$ \\ Published online: 8 May 2019 \\ (C) International Association of Medical Science Educators 2019
}

We read with great interest the recent article by L. J. Van Scoy et al. (1) about introducing a 'mini-curriculum' to help medical students around the subject of end-of-life discussions and we thoroughly commend the aims of the suggested course. As medical students in our penultimate year at the Imperial College School of Medicine, we reflected on our knowledge and confidence around having these conversations and how our teaching and experiences have impacted this.

The game proposed in the course appears a good trigger for students to start openly talking about death. However, having a game where people can 'opt not to share' would, in our experience, lead to social loafing. Conversely, as mentioned in the qualitative analysis, 'forced revealing' is something that could negatively impact confidence. Feedback was also taken immediately after the game was played. This perhaps did not allow sufficient reflection, which could explain the increase in confidence between the 2 nd and 3rd questionnaire, which was then aided by the reflective essay.

In the qualitative analysis, it mentions the lack of 'clinical connections'. It is hard for students to see how the content is relevant when it is not in a clinical scenario. In our opinion, the second year of medical school could be quite early to be assessing for confidence given the potential lack of clinical knowledge. This could be a confounding factor in some questions where there was not much improvement in student confidence.

Emma Mittelman

emma.mittelman14@imperial.ac.uk

Rajiv Ark

rajiv.ark14@imperial.ac.uk

1 Imperial College London, South Kensington Campus, London SW7 2AZ, UK
Although confidence was the primary outcome measured, we feel it is important to know that these sessions are improving our knowledge in the area and it would be interesting to see how the 'mini-curriculum' influenced performance in exams or OSCEs (Objective Structured Clinical Examinations).

We have had quite sparse and varied teaching on end-oflife planning and conversations. During our Oncology and Palliative Care placement this year, we had a communication seminar specific to 'difficult conversations'. We found these practice scenarios most helpful, allowing us to make mistakes and try out different phrases as well as receive constructive feedback. From our experience, we would urge for more practical experience at 'initiating', 'contributing' and 'leading' end-of-life conversations being added to the course. This would also help to ensure that the first time these conversations are tried is not with a patient or their family, helping students to feel more ready to confront such a daunting aspect of being a doctor.

Yours sincerely, Emma Mittelman and Rajiv Ark Imperial College London, London, UK emma.mittelman14@imperial.ac.uk and rajiv.ark14@imperial.ac.uk

\section{Reference}

1. Van Scoy LJ, Green MJ, Volpe R. Evaluating an advance care planning curriculum: a lecture, a game, a patient, and an essay. Medical Science Educator. 2019:1-10.

Publisher's Note Springer Nature remains neutral with regard to jurisdictional claims in published maps and institutional affiliations. 